\title{
Effect of the pat, fk, stpk Gene Knock-out and $m d h$ Gene Knock-in on Mannitol Production in Leuconostoc mesenteroides
}

\author{
Peng Yu-Wei and Jin Hong-Xing* \\ School of Chemical Engineering and Technology, Hebei University of Technology, Tianjin 300130, P.R. China
}

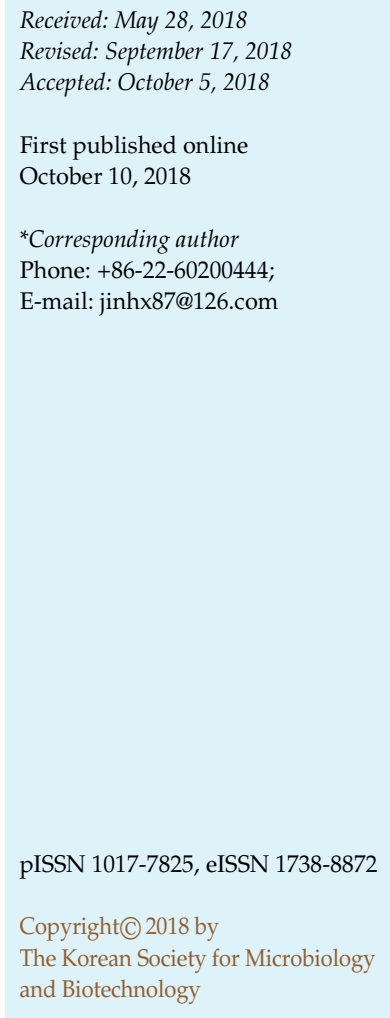

\begin{abstract}
Leuconostoc mesenteroides can be used to produce mannitol by fermentation, but the mannitol productivity is not high. Therefore, in this study we modified the chromosome of Leuconostoc mesenteroides by genetic methods to obtain high-yield strains for mannitol production. In this study, gene knock-out strains and gene knock-in strains were constructed by a two-step homologous recombination method. The mannitol productivity of the pat gene (which encodes phosphate acetyltransferase) deletion strain ( $\Delta$ pat::amy), the $f k$ gene (which encodes fructokinase) deletion strain $(\Delta f k:: a m y)$ and the stpk gene (which encodes serine-threonine protein kinase) deletion strain ( $\Delta$ stpk::amy) were all increased compared to the wild type, and the productivity of mannitol for each strain was $84.8 \%, 83.5 \%$ and $84.1 \%$, respectively. The mannitol productivity of the $m d h$ gene (which encodes mannitol dehydrogenase) knock-in strains ( $\Delta p a t:: m d h, \Delta f k:: m d h$ and $\Delta s t p k:: m d h)$ was increased to a higher level than that of the single-gene deletion strains, and the productivity of mannitol for each was $96.5 \%, 88 \%$ and 93.2\%, respectively. The multi-mutant strain $\Delta d t s \Delta l d h \Delta p a t:: m d h \Delta s t p k:: m d h \Delta f k:: m d h$ had mannitol productivity of $97.3 \%$. This work shows that multi-gene knock-out and gene knock-in strains have the greatest impact on mannitol production, with mannitol productivity of $97.3 \%$ and an increase of $24.7 \%$ over wild type. This study used the methods of gene knock-out and gene knock-in to genetically modify the chromosome of Leuconostoc mesenteroides. It is of great significance that we increased the ability of Leuconostoc mesenteroides to produce mannitol and revealed its broad development prospects.
\end{abstract}

Keywords: Leuconostoc mesenteroides, mannitol productivity, gene knock-out, gene knock-in

\section{Introduction}

Mannitol, a C6 sugar alcohol, is widely used in the chemical, medical and food industries [1]. At present, industrial production of mannitol usually occurs through catalytic hydrogenation [2]. However, this production method requires high pressure at a high temperature with the poor choice of Raney nickel as catalyst [3]. If a 50/50 glucose-fructose mixture is used as the substrate, a 25/75 mannitol-sorbitol mixture will be obtained [4]. Moreover, it is relatively difficult to separate sorbitol and mannitol, which results in even higher production costs and decreased yields [5].

The mannitol content in phaeophyta reaches only as much as $10 \%-20 \%$ and it can be extracted by water recrystallization, ethanol extraction or electrodialysis [6]. Although the extraction method can be used to obtain mannitol, the production cost is relatively high, and the extraction process generates a large amount of waste water and pollutes the environment.

Mannitol can also be produced by enzyme conversion and microbial fermentation [7]. Enzymatic production of mannitol from fructose with mannitol dehydrogenase is also possible [8]. Furthermore, this reaction requires a high-priced co-factor such as $\mathrm{NAD}(\mathrm{P}) \mathrm{H}$ that also needs to be regenerated [9].

It is well known that many microorganisms in nature can synthesize mannitol, such as yeast, mold and some bacteria strains have mannitol production capacity [7]. Tomaszewska, L. et al. [10] used the Yarrowia lipolytica yeast to produce 
mannitol, but the fermentation period is 10 days. The long fermentation period of yeast and mold resulted in lower rate of productivity [6]. Homo-fermentative LAB can produce mannitol, but at very low levels [5]. Mainly heterofermentative lactic acid bacteria also produce mannitol without the co-formation of sorbitol [11].

Leuconostoc mesenteroides, a group of hetero-fermentative lactic acid bacteria, is a gram-positive bacterium and aerotolerant anaerobe. The chromosome genome of Leuconostoc mesenteroides is about $2 \mathrm{M}$ (https://www.ncbi. nlm.nih.gov/genome/- genomes/1078), and the metabolic

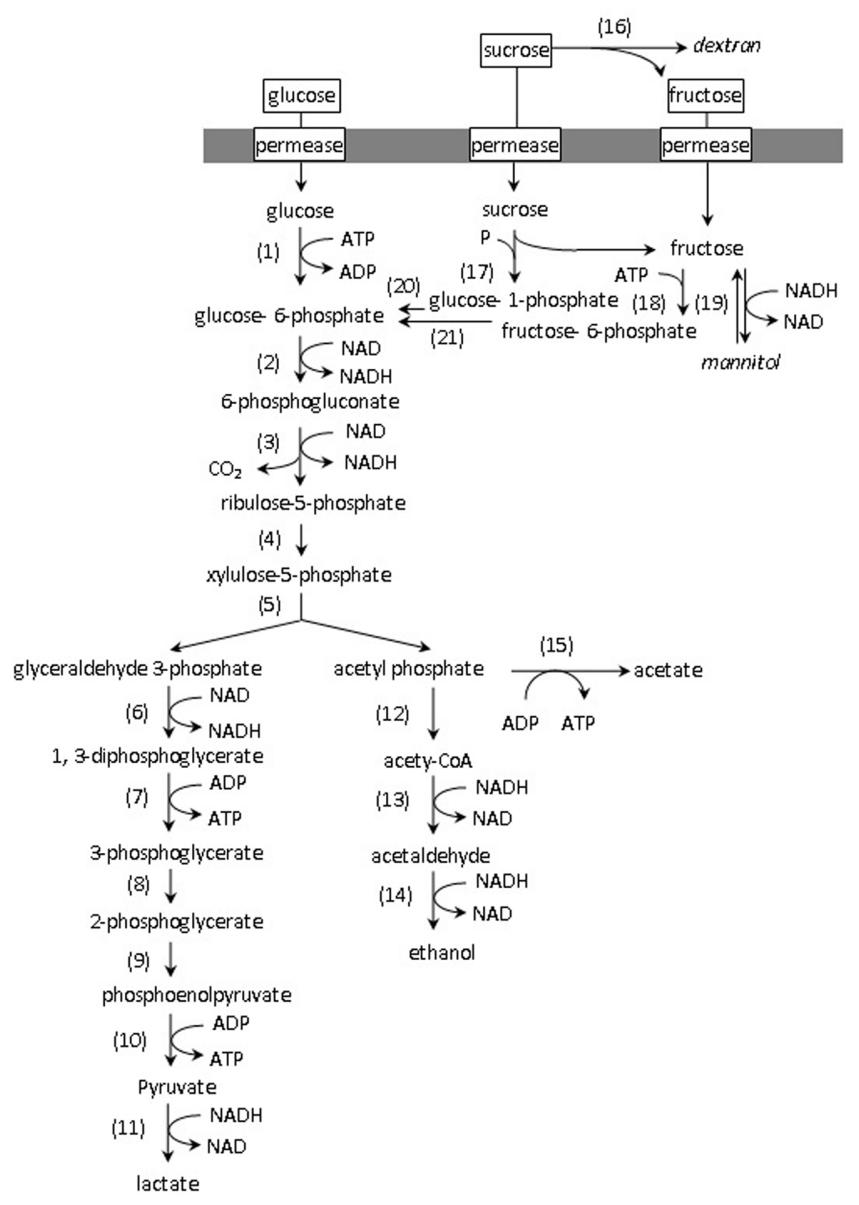

Fig. 1. The main carbon metabolic pathways in L. mesenteroides. (1) glucokinase, (2) glucose-6-phosphate dehydrogenase, (3) 6phosphogluconate dehydrogenase, (4) ribulose-5-phosphate 3-epimerase, (5) phosphoketolase, (6) glyceraldehyde 3-phosphate dehydrogenase, (7) phosphoglycerate kinase, (8) phosphoglycerate mutase, (9) enolase, (10) pyruvate kinase, (11) lactate dehydrogenase, (12) phosphotransacetylase, (13) aldehyde dehydrogenase, (14) alcohol dehydrogenase, (15) acetate kinase, (16) dextransucrase, (17) sucrose phosphorylase, (18) fructokinase, (19) mannitol dehydrogenase, (20) phosphoglucose mutase, (21) phosphoglucose isomerase. pathway is uncomplicated and the fermentation period of Leuconostoc mesenteroides is shorter than that of yeast [12]. Simplicity of separation and purification of mannitol is a process in which the small, white needle-like crystals of mannitol appeared upon refrigeration of the cell-free fermentation broth at $4^{\circ} \mathrm{C}$ [13]. Mannitol can be produced (Fig. 1) when Leuconostoc are fermented with fructose and glucose [14], or only with fructose [15] or sucrose [16]. In order to reduce fermentation costs, Fontes CP. et al. [17] used sucrose as a substrate. However, sucrose, under the action of dextransucrase, can be converted to dextran to reduce mannitol productivity [18]. Therefore, Zhang et al. [19] inactivated the $d t s$ gene which encodes the dextransucrase, resulting in a decrease of dextran in the mutant by $28.8 \%$ and an increase in the mannitol yield of $15.3 \%$.

In order to increase mannitol productivity, in this study the $f k$, pat, and stpk genes (which encode the fructokinase, phosphate acetyltransferase and serine-threonine protein kinase respectively) were knocked out and the $m d h$ gene (which encodes the mannitol dehydrogenase) was knocked in.

\section{Materials and Methods}

\section{Microorganisms and Medium}

The bacterial strains used in this study are listed in Table 1. E. coli $\mathrm{DH} 5 \alpha$ was grown in LB broth or on agar plates at $37^{\circ} \mathrm{C}$ and supplemented with $100 \mathrm{mg} / 1$ ampicillin. L. mesenteroides CGMCC1.10327 and L. mesenteroides ATCC8293 were grown in MRS medium [19] or on agar plates of MRS at $30^{\circ} \mathrm{C}$, with added trypan blue and soluble starch [20].

\section{PCR Amplification of DNA}

The chromosomal DNA of Leuconostoc can be extracted by the method of Vingataramin et al [21]. The plasmids and primers used in this study are listed in Tables 2 and 3, respectively.

The $f k$ fragment, pat fragment and stpk fragment were amplified by PCR from L. mesenteroides CGMCC1.10327 chromosomal DNA using oligonucleotides pat-u/pat-d, fk-u/fk-d and stpk-u/stpk-d (Table 2) as primers, respectively. The $\alpha$-amylase expression cassette $(\alpha$-amy) was amplified by PCR from pTA2-amy using oligonucleotides amy-u/amy-d (Table 2) as primers. The $m d h$ expression cassette was amplified by PCR from L. mesenteroides ATCC8293 chromosomal DNA using oligonucleotides mdh-u/ mdh-d (Table 2) as primers. The $f k$ fragment, stpk fragment and pat fragment were digested with the restriction endonuclease (restriction sites were introduced by primers) and inserted into similarly digested pUC19, resulting in pUC19-fk, pUC19-pat and pUC19-stpk, respectively. 
Table 1. Strains used in this study.

\begin{tabular}{|c|c|c|}
\hline Strains & Relevant characteristics & Source reference \\
\hline E. coli DH5 $\alpha$ & 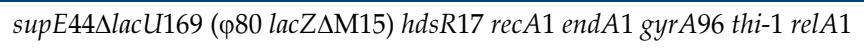 & TaKaRa \\
\hline CGMCC1.10327 & Wild type L. mesenteroides & This laboratory \\
\hline ATCC 8293 & Wild type L. mesenteroides & This laboratory \\
\hline spat::amy & CGMCC1.10327 with knock-out pat and knock-in amy & This work \\
\hline$\Delta f k:: a m y$ & CGMCC1.10327 with knock-out $f k$ and knock-in amy & This work \\
\hline$\Delta s t p k:: a m y$ & CGMCC1.10327 with knock-out stpk and knock-in amy & This work \\
\hline$\Delta p a t:: m d h$ & CGMCC1.10327 with knock-out pat and knock-in $m d h$ & This work \\
\hline$\Delta f k:: m d h$ & CGMCC1.10327 with knock-out $f k$ and knock-in $m d h$ & This work \\
\hline$\Delta s t p k:: m d h$ & CGMCC1.10327 with knock-out stpk and knock-in $m d h$ & This work \\
\hline$\Delta$ stpk & CGMCC1.10327 with knock-out stpk & This work \\
\hline$\Delta p a t:: m d h \Delta s t p k:: m d h$ & CGMCC1.10327 with knock-out pat, stpk and knock-in $m d h$ & This work \\
\hline$\Delta p a t:: m d h \Delta f k:: m d h$ & CGMCC1.10327 with knockout $p a t, f k$ and knockin $m d h$ & This work \\
\hline$\Delta f k:: m d h \Delta s t p k:: m d h$ & CGMCC1.10327 with knock-out $f k$, stpk and knock-in $m d h$ & This work \\
\hline$\Delta p a t:: m d h \Delta s t p k:: m d h-\Delta f k:: m d h$ & CGMCC1.10327 with knock-out pat, stpk, fk and knock-in $m d h$ & This work \\
\hline$\Delta d t s \Delta l d h \Delta p a t:: m d h-\Delta s t p k:: m d h \Delta f k:: m d h$ & CGMCC1.10327 with knock-out $d t s, l d h, p a t, s t p k, f k$ and knock-in $m d h$ & This work \\
\hline$\Delta$ pat::amy/pat & pCW7-pat transformated into $\Delta$ pat::amy & This work \\
\hline$\Delta f k:: a m y / f k$ & pCW7-fk transformated into $\Delta f k:: a m y$ & This work \\
\hline$\Delta s t p k:: a m y / s t p k$ & pCW7-stpk transformated into $\Delta$ stpk::amy & This work \\
\hline
\end{tabular}

\section{Construction of Homologous Recombination Vector}

The up-stream and down-stream flanking regions of the gene were amplified by PCR from pUC19-fk, pUC19-pat and pUC19stpk separately. Both flanking regions were then spliced by overlap extension PCR (KpnI was introduced by primers). These PCR products were digested with EcoRI and HindIII and subsequently ligated to similarly digested pUC19, resulting in homologous recombination plasmids without a marker gene. Then the $\alpha$-amy expression cassette was digested with KpnI and inserted into the homologous recombination plasmids, resulting in homologous recombination plasmids which carried an $\alpha$-amylase marker gene. In the same way, the $m d h$ expression cassette was inserted into the homologous recombination plasmids, resulting in homologous recombination plasmids which carried the $m d h$ expression cassette.

\section{Construction of Mutant Strains and Complement Strains}

Competent cells of $L$. mesenteroides can be prepared by the method of Zhang et al. [19]. A fresh culture of strain CGMCC1.10327 was inoculated into MRS broth containing ampicillin (final concentration of $0.48 \mathrm{mg} / \mathrm{l}$ ), and cells were cultured to reach an $\mathrm{OD}_{660}$ of 0.5. Cells were harvested and pre-treated with LiAc-DTT (100 mmol/1 LiAc, $10 \mathrm{mmol} / 1 \mathrm{DTT}, 0.5 \mathrm{~mol} / 1$ sucrose, $10 \mathrm{mmol} / \mathrm{l}$ Tris-HCl) supplemented with $100 \mathrm{U} / \mathrm{ml}$ lysozyme for $20 \mathrm{~min}$. After pre-treatment, PBS ( $1 \mathrm{mmol} / 1 \mathrm{KH}_{2} \mathrm{PO}_{4}-\mathrm{K}_{2} \mathrm{HPO}_{4}, 1 \mathrm{mmol} / 1$ $\mathrm{MgCl}_{2}, 0.5 \mathrm{~mol} / 1$ sucrose) of $\mathrm{pH} 6.9$ was used to wash and resuspend the cells. Next, competent cells were mixed with transforming DNA in a microfuge tube, transferred to cold electroporation cuvettes and placed on ice for $5 \mathrm{~min}$. A pulse was applied under the following condition: $1,400 \mathrm{~V}, 25 \mu \mathrm{F}, 300 \Omega$ and $4 \mathrm{~ms}$. The cells were immediately resuspended in $1 \mathrm{ml}$ MRS broth containing $2 \%$ sucrose and incubated for $3 \mathrm{~h}$ at $30^{\circ} \mathrm{C}$. The electropulsed strains were diluted $10^{7}$ on MRS agar plates incubated at $30^{\circ} \mathrm{C}$ for 5 days, in which trypan blue and soluble starch were added.

In this study, the shuttle vector pCW7 was used, which was constructed by Tian et al. [20], to construct the complementary vector. The expression cassettes of pat, $f k$, and stpk were amplified by PCR from chromosomal DNA of L. mesenteroides CGMCC1.10327 respectively. These expression cassettes were then inserted into the shuttle vector pCW7. Afterwards, the complementary vectors were introduced into the single-gene deletion strains by electric transformation.

\section{Verification of Mutant Strains}

The pat, $f k$, and stpk fragments were amplified by PCR from the chromosome DNA of single mutant strains and wild-type strains, respectively. The sizes of fragments were compared with a marker by agarose gel electrophoresis.

\section{Mannitol Assay}

Mutant strains, complement strains and wild-type strains were incubated in liquid fermentation medium. Cultivated for $20 \mathrm{~h}$, the concentrations of mannitol were measured by colorimetric 
Table 2. Primers used in this study.

\begin{tabular}{|c|c|}
\hline Name & Sequences \\
\hline pat-u & TGTGAATTCTTTTGCTAAGCCTTGT (EcoRI) \\
\hline pat-d & TGTGAATTCGTGAAGATCCCCGTAT (EcoRI) \\
\hline $\mathrm{fk}-\mathrm{u}$ & ACGAAGCTTGAAGCAGGTGGAACGA (HindIII) \\
\hline fk-d & TGTGAATTCTAGCAACGGGTACGAT (EcoRI) \\
\hline stpk-u & ACGGAATTCTCCTCACTACTTGTTG (EcoRI) \\
\hline stpk-d & ACGGAATTCATGATGCGTCTTGATA (EcoRI) \\
\hline amy-u & TTGGTACCTTTGGCGTGATTATCAG (KpnI) \\
\hline amy-d & TTGGTACCCGAAGGTGAAGTTATAG (KpnI) \\
\hline mdh-u & ATGGTACCATTATGCCTCTTCGCCG (KpnI) \\
\hline mdh-d & ATGGTACCCACGTGATACTGTTGTC (KpnI) \\
\hline patl-u & CGGAATTCTCGACTTATAATGCTTG (EcoRI) \\
\hline patl-d & TAGGATCCTAGGTACCAAGCGAAGAGCGTTATGT (KpnI) \\
\hline patr-u & TTGGTACCTAGGATCCTACTTCGCCTTTTTGCAT (KpnI) \\
\hline part-d & CGAAGCTTAGGCATTTATGGAACTT (HindIII) \\
\hline $\mathrm{fkl-u}$ & CGGAATTCTTGCGGATCAC (EcoRI) \\
\hline fkl-d & TTGGTACCGCGCCATTAACGTCAGT (KpnI) \\
\hline $\mathrm{fkr}-\mathrm{u}$ & AATGGCGCGGTACCAAAAGCATCCT (KpnI) \\
\hline fkr-d & GCAAGCTTCAGCAAAACTT (HindIII) \\
\hline stpkl-u & CAGGAATTCTGTTGAACTGCTTGAGG (EcoRI) \\
\hline stpkl-d & GTCGGATCCGGTACCAGCGTCAGATAGTGTA (KpnI) \\
\hline stpkr-u & GCTGGTACCGGATCCGACCCAACATAATCTC (KpnI) \\
\hline stpkr-d & CGCAAGCTTTGTTGACCGGACACCTA (HindIII) \\
\hline patc-u & GA $\underline{\text { AGATCTCGATTCCTGTTATCCGCAT (BgIII) }}$ \\
\hline patc-d & GAAGATCTCGTGGCTTTTTTGGAAGTC (BgIII) \\
\hline fkc-u & GAAGATCTCAGATATTATTGAAGTGTT (BglII) \\
\hline fkc-d & GAAGATCTTGAAAATTAAAGTAATGTT (BglII) \\
\hline stpkc-u & GCGTCGACTTTAGACACGTTGTTATTG (SalI) \\
\hline stpkc-d & GCGTCGACTGACGAAAAAGTTGTGATT (SalI) \\
\hline paty-u & ACATTCTCTTCATTGGCTC \\
\hline paty-d & GACTTTATGGAACTTTTTG \\
\hline fky-u & ACTCAGTAGAGCAAGTCAT \\
\hline fky-d & TATCAGGGCGTAAAATCAT \\
\hline stpky-u & GAACTGCTTGAGGAACTAC \\
\hline stpky-d & GACCGGACACCTAATTATG \\
\hline
\end{tabular}

determination [19]. To $1 \mathrm{ml}$ of the fermentation supernatant, we added $1.5 \mathrm{ml}$ concentrated hydrochloric acid, and this was heated in a boiling water bath for $10 \mathrm{~min}$ (remove the interference of fructose), cooled, diluted, combined with $1 \mathrm{ml}$ sodium periodate solution $(0.015 \mathrm{~mol}$ sodium periodate dissolved in $0.12 \mathrm{~mol} / \mathrm{l}$ hydrochloric acid solution) and mixed. After letting the mixture sit at room temperature for $10 \mathrm{~min}$, we added $2 \mathrm{ml} 0.1 \% \mathrm{~L}$ rhamnose solution, and then mixed and added $4 \mathrm{ml}$ freshly prepared Nash reagent $(75 \mathrm{~g}$ acetic acid, $1 \mathrm{ml}$ glacial acetic acid and $1 \mathrm{ml}$ acetonitrile, dilute to $500 \mathrm{ml}$ ). The mixture was heated in a $53^{\circ} \mathrm{C}$ water bath for 15 min (producing yellow 3, 5- diacetyl-1,4dehydrodimethylpyridine, which has a large absorption at $412 \mathrm{~nm}$ ), and cooled to room temperature. The absorbance was measured at $412 \mathrm{~nm}$.

\section{Results}

\section{Construction of Homologous Recombination Vector}

In this study, homologous recombinant vectors were constructed by overlap extension PCR. The up-stream and down-stream flanking regions of the pat were amplified by PCR from pUC19-pat, using primer paris patl-u/patl-d, part-u/part-d (KpnI was added to the complementary region of the primer downstream of the left homology fragment and the upstream primer of the right homology fragment), resulting in homologous recombinant vector pUC19-pat. In the same way, it resulted in pUC19-stpk and pUC19-fk too. Then the $\alpha$-amy expression cassette was digested with $\mathrm{KpnI}$ and inserted into the homologous recombination vectors, resulting in homologous recombinant vectors pUC19-pat::amy, pUC19-fk::amy and pUC19stpk::amy, respectively. With the same method, the $m d h$ expression cassette was inserted into the homologous recombination vectors, resulting in homologous recombinant vectors pUC19-pat::mdh, pUC19-fk::mdh and pUC19stpk::mdh, respectively.

\section{Single Gene Deficiency of $L$. mesenteroides}

Acetyl phosphate can be converted into acetyl-coenzyme A to produce ethanol with the phosphate acetyltransferase (pat) in PPK pathway [22]. If the pat gene is knocked out, it is possible for the procedure to be blocked, and the mixture accumulates more NADH for mannitol production and allows more carbon sources to flow to mannitol production. Jin et al. (China patent CN 106754555A) showed that in the Leuconostoc genome, there are other genes that function similarly to the acetaldehyde dehydrogenase gene, so there is a portion of ethanol produced when the aldehyde dehydrogenase gene is knocked out. In this study, the homologous recombinant vector pUC19-pat::amy was introduced into L. mesenteroides CGMCC1.10327 by the method of electric transformation (Fig. 2A), resulting in the single-gene deletion strain $\Delta$ pat::amy.

Fructose can be converted into 6-phosphoric acid fructose by fructokinase $(f k)$, whereupon it then enters the PPK pathway to produce lactic acid and ethanol [23]. The hypothesis of the present work is that inactivation of the $f k$ gene would prevent the leakage of fructose into the PPK 
Table 3. Plasmids used in this study.

\begin{tabular}{|c|c|c|}
\hline Plasmids & Relevant characteristics & Source or reference \\
\hline pUC19 & $A m p^{R}$ & TaKaRa \\
\hline pUC19-pat & pUC19 containing the gene of pat & This work \\
\hline pUC19-fk & pUC19 containing the gene of $f k$ & This work \\
\hline pUC19-pat::amy & pUC19 containing $\alpha$-amy upstream and downstream fragment of pat, $\mathrm{Amp}^{\mathrm{R}}$ & This work \\
\hline pUC19-fk::amy & pUC19 containing $\alpha$-amy upstream and downsteam fragment of $f k, \mathrm{Amp}^{\mathrm{R}}$ & This work \\
\hline pUC19-stpk::amy & pUC19 containing $\alpha$-amy upsteam and downsteam fragment of stpk, $\mathrm{Amp}^{\mathrm{R}}$ & This work \\
\hline pUC19-pat::mdh & pUC19 containing $m d h$ upsteam and downsteam fragment of pat, $A m p^{\mathrm{R}}$ & This work \\
\hline pUC19-fk::mdh & pUC19 containing $m d h$ upsteam and downsteam fragment of $f k, \mathrm{Amp}^{\mathrm{R}}$ & This work \\
\hline pUC19-stpk::mdh & pUC19 containing $m d h$ upsteam and downsteam fragment of $s t p k, \mathrm{Amp}^{\mathrm{R}}$ & This work \\
\hline pTA2-amy & pTA2 containing amy & The lab \\
\hline pCW7 & pCW4 containing the gene of $a p r, a m y$, and oriT, $\mathrm{Amp}^{\mathrm{R}}$ & The lab \\
\hline pCW7-fk & pCW7 containing the expression cassettes of $f k, \mathrm{Amp}^{\mathrm{R}}$ & This work \\
\hline pCW7-pat & pCW7 containing the expression cassettes of pat, $\mathrm{Amp}^{\mathrm{R}}$ & This work \\
\hline pCW7-stpk & pCW7 containing the expression cassettes of $s t p k, \mathrm{Amp}^{\mathrm{R}}$ & This work \\
\hline
\end{tabular}

pathway and give improved yield of mannitol from fructose. In this study, the homologous recombinant vector pUC19-fk::amy was introduced into L.mesenteroides CGMCC1.10327 by the method of electric transformation, resulting in the single-gene deletion strain $\Delta f k:: a m y$.

Serine threonine protein kinase (stpk) is generally considered to exist in the eukaryotic, but it also exists in some bacteria [24]. Proteome analysis of producing dextran and non-producing dextran showed that the association with the two-component system of serine threonine protein kinase was significantly raised when dextran was produced [25]. The hypothesis of the present work is that knock-out of the stpk gene would hinder the processing and secretion of dextransucrase, resulting in more conversion of sucrose
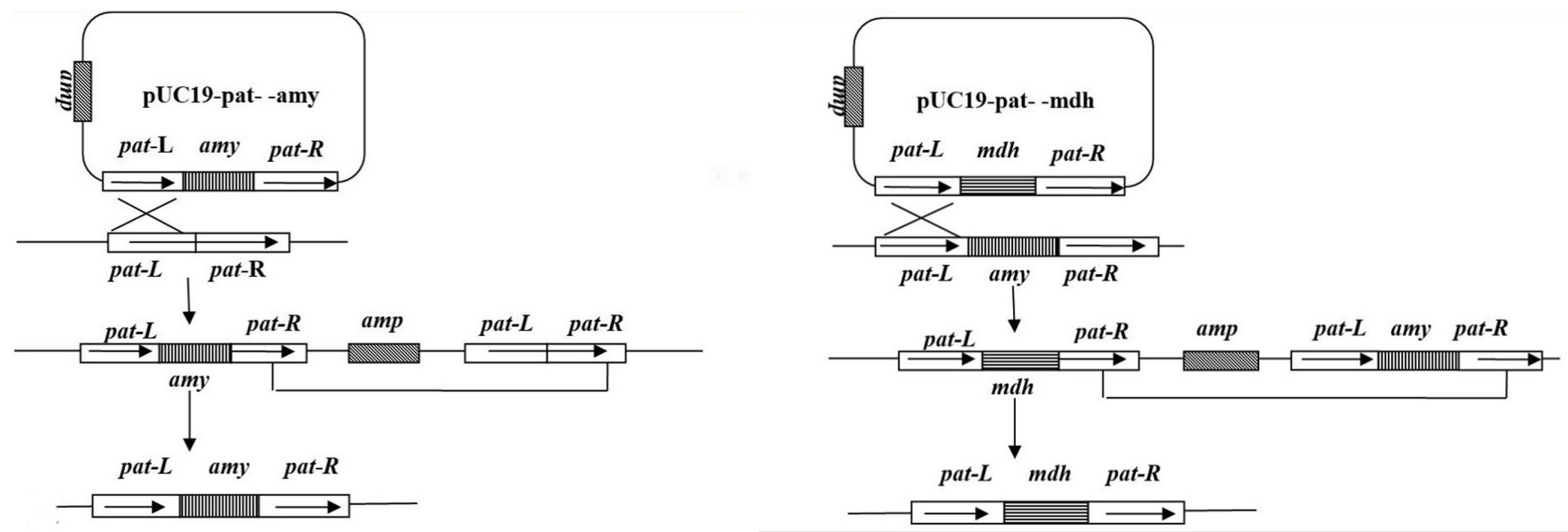

A

B

Fig. 2. Construction of a disruption in the L. mesenteroides pat gene and knock-in mdh gene.

A two-step homologous recombination method was used to knock out the pat gene and knock in the mdh gene. Integration of this plasmid into the chromosome can take place via the pat-L region. This integration results in both a truncated and a disrupted copy of the pat gene. On the other hand, disrupted and intact copies of the gene are obtained when integration occurs via the pat- $\mathrm{R}$ region. A: Integration of this homologous recombination fragment which carried the amy gene into the chromosome DNA of L. mesenteroides. B: Integration of this homologous recombination fragment which carried the mdh gene into the chromosome DNA of L. mesenteroides. 


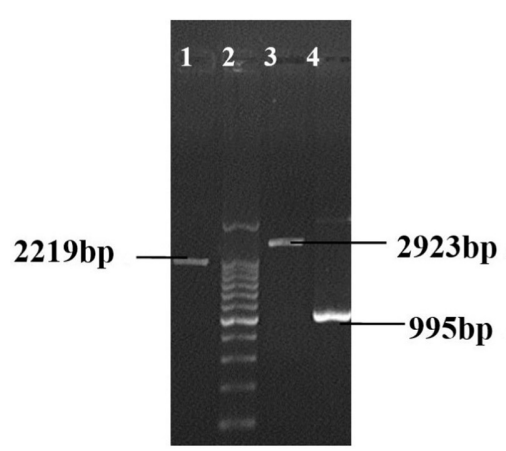

A

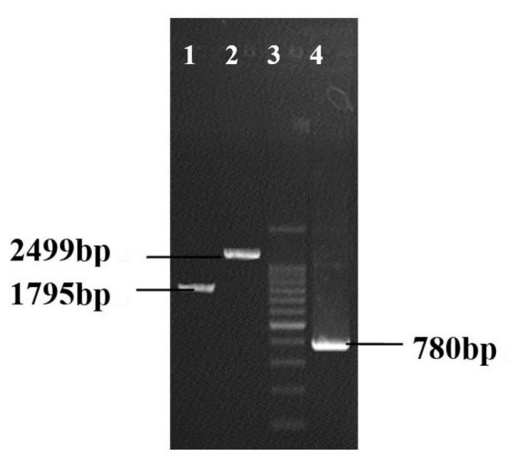

B

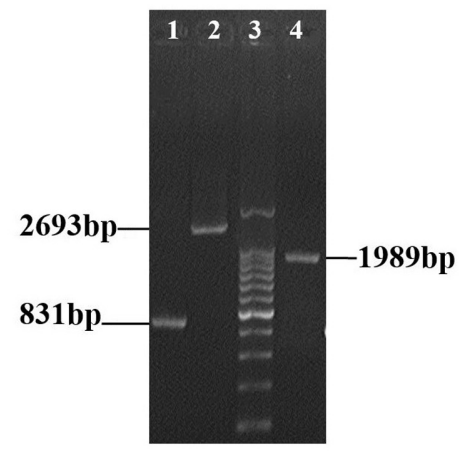

C

Fig. 3. The conformation of pat, fk, stpk inactivation and mdh expression mutant strains by PCR anylasis: M, DNA marker, 1 , spat::mdh, 2, spat::amy, 4, $\Delta \mathrm{fk}:: \mathrm{mdh}, 5$, $\Delta \mathrm{fk}:$ :amy, 8, $\Delta$ stpk::amy, 9, $\Delta$ stpk::mdh, 3, 6, 7, PCR product of pat, fk, stpk, from CGMCC1.10327, respectively.

to fructose, thus increasing mannitol productivity. In this study, the homologous recombinant vector pUC19-stpk::amy was introduced into the L. mesenteroides CGMCC1.10327 by the method of electric transformation, resulting in the single gene-deletion strain $\Delta$ stpk::amy.

The single-gene deletion strain $\Delta p a t:: a m y$ was verified by PCR using the primer paty-u/paty-d and the chromosomal DNA of $\Delta p a t:: a m y$ as a template. Similarly, $\Delta f k:: a m y$ and $\Delta s t p k:: a m y$ were verified by PCR using the primers fky-u/ fky-d and stpky-u/stpky-d with the chromosomal DNA of $\Delta f k:: a m y$ and $\Delta s t p k:: a m y$ as a template, respectively. The sizes of pat, $f k$ and stpk homologous recombination fragments with $\alpha$-amy expression cassette were 2,293 bp, 2,499 bp, and 2,693 bp, respectively. The resultant (Fig. 3) gave the expected fragments in similar PCR tests. Thus, these mutants have the correct structures.

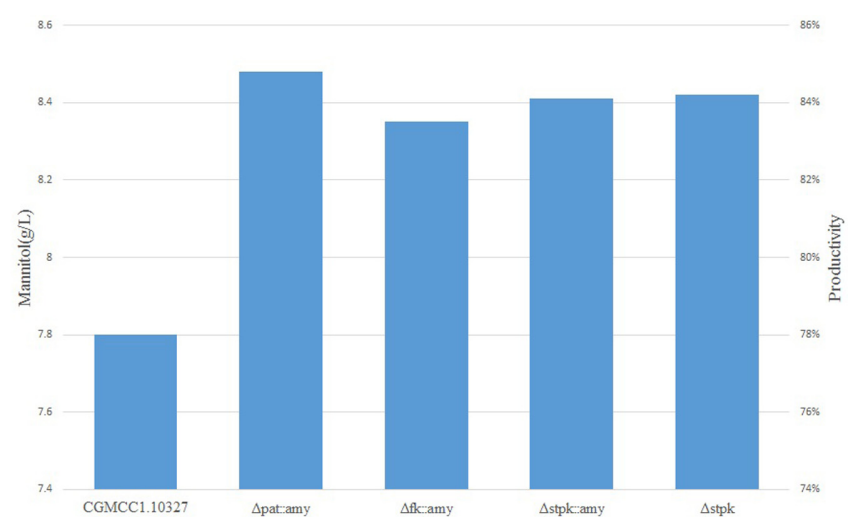

Fig. 4. Mannitol (g/l) analysis of sucrose fermentation by wild-type and mutant strains.

\section{Effect of Mutants on Mannitol Production}

Yield of fermentation products of mannitol was measured and tabulated (Fig. 4) for batch culture of single-gene deletion strains $\Delta p a t:: a m y, \Delta f k:: a m y, \Delta s t p k:: a m y$ and wildtype strain. The study found that the accumulation of mannitol reached the maximum when the fermentation period was about $20 \mathrm{~h}$. After $20 \mathrm{~h}$, the mannitol decomposed and was reused, resulting in decreased accumulation of mannitol (Mannitol is weakly used) [26]. The experimental results also showed that the mannitol synthesis ability of each single-gene deletion strain was increased compared with the wild-type strain.

The study showed that the pat gene was deleted from a single mutation, resulting in the mannitol productivity reaching $84.8 \%$ (an increase of $8.71 \%$ over the wild-type strain) and almost no detectable ethanol (ethanol productivity

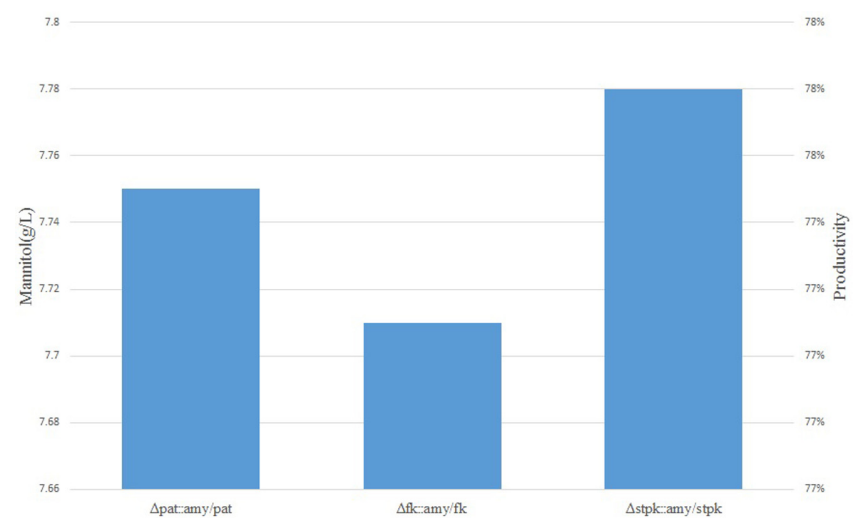

Fig. 5. Mannitol (g/l) analysis of sucrose fermentation by wild-type and mutant strains. 


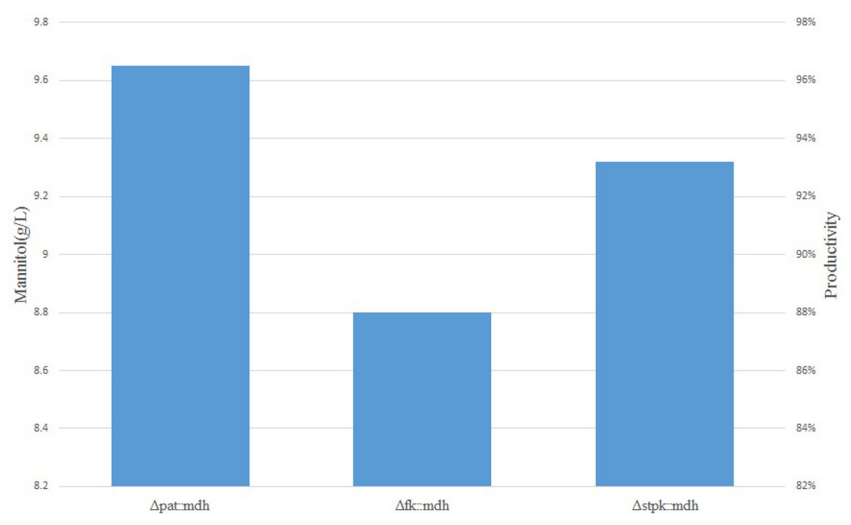

Fig. 6. Mannitol $(\mathrm{g} / \mathrm{l})$ analysis of sucrose fermentation by wild-type and mutant strains.

is not listed in the Figs. 4-8), so it is far more influential than inactivation of $f k$ or stpk.

\section{Construction of Complement Strains}

The shuttle vector pCW7-pat was introduced into the $\Delta$ at::amy by the method of electric transformation, resulting in complement strain $\Delta$ pat::amy/pat. With the same method, the complement strains $\Delta f k:: a m y / f k$ and $\Delta s t p k:: a m y / s t p k$ were constructed. The yield of the fermentation products of mannitol was measured and tabulated (Fig. 5). There was no significant difference in mannitol production between the complement strains and wild type. It is confirmed that inactivation of pat, $f k$, and stpk resulted in increased mannitol productivity.

\section{Knock-in mdh of L. mesenteroides}

L. mesenteroides can be used with mannitol dehydrogenase

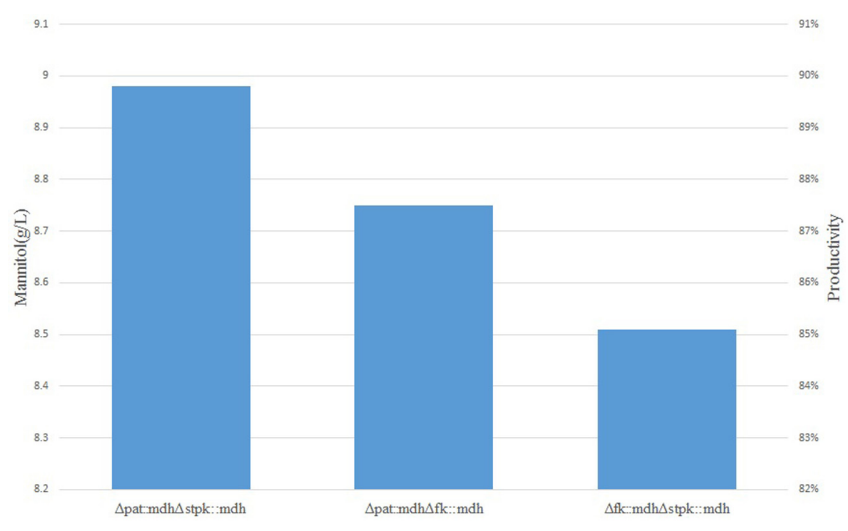

Fig. 7. Mannitol $(\mathrm{g} / \mathrm{l})$ analysis of sucrose fermentation by wild-type and mutant strains.

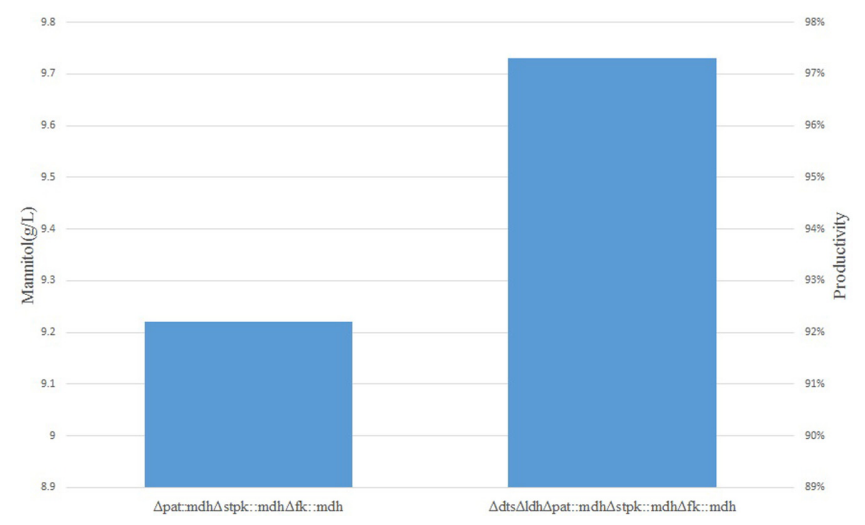

Fig. 8. Mannitol (g/l) analysis of sucrose fermentation by wild-type and mutant strains.

to convert fructose to mannitol. In order to obtain a highyielding mannitol-producing strain, the $m d h$ expression cassette was inserted into the homologous recombination vectors in this study, resulting in pUC19-pat::mdh, pUC19fk::mdh and pUC19-stpk::mdh, respectively. Then it integrated into the chromosomal DNA of L. mesenteroides CGMCC1.10327 by two-step homologous recombination (Fig. 2B).

The yield of the fermentation products of mannitol was measured and tabulated (Fig. 6). According to this production, the productivity of mannitol was increased so it's better than the single-gene deletion strains. The singlegene-deleted and $m d h$ knock-in strain $\Delta p a t:: m d h$ had the highest mannitol productivity reaching $96.5 \%$, an increase of $23.7 \%$ over the wild-type strain.

The knock-in strains were verified by the same method as the single-gene deletion strains. The sizes of pat, $f k$, and stpk homologous recombination fragments with $m d h$ expression cassette were 2,219 bp, 1,795 bp, and 1,989 bp, respectively. The results (Fig. 3 ) gave the expected fragments as shown in similar PCR tests. Thus, these knock-in strains have the correct structures.

\section{Double Gene Deficiency and Gene Knock-in of L. mesenteroides}

In order to further study the effect of phosphate acetyltransferase, fructokinase, serine-threonine protein kinase and mannitol dehydrogenase on the production of mannitol by L. mesenteroides CGMCC1.10327, double-gene deficiency and $m d h$ gene knock-in strains were constructed. Firstly, the homologous recombinant vector pUC19-fk::amy was introduced into the $\Delta p a t:: m d h$ by electric transformation, resulting in double-gene deficiency strain $\Delta p a t:: m d h \Delta f k:: a m y$. 
Then, the homologous recombinant vectors pUC19-fk::mdh were introduced into the $\Delta p a t:: m d h \Delta f k: a m y$ by electric transformation, resulting in double-gene deficiency and $m d h$ gene knock-in strain $\Delta p a t:: m d h \Delta f k: m d h$. Similarly, the $\Delta p a t:: m d h \Delta s t p k:: m d h$ and $\Delta f k:: m d h \Delta s t p k:: m d h$ were constructed respectively.

The yield of the fermentation products of mannitol was measured and tabulated (Fig. 7). The deficiency of both pat and stpk in the double-gene deficiency strain was the most significant, and the productivity of mannitol was $89.8 \%$ (an increase of $15.1 \%$ over the wild-type strain).

\section{Multi-Gene Deficiency and Gene Knock-in of $L$. mesenteroides}

Leuconostoc can be used to produce dextran [27] and as lactic acid bacteria, its most important fermentation product is lactic acid. In order to reduce the effect of lactic acid and dextran on mannitol production, Zhang et al. [19] knocked out the $d t s$ (dextransucrase) gene and the mannitol productivity was increased by $15.3 \%$ with the wild strain. Tian et al. [20] knocked out the ldh (lactate dehydrogenase) gene and the mannitol productivity was increased by $7 \%$ with the wild strain. In this study, the multi-gene deficiency and $m d h$ gene knock-in strains $\Delta p a t:: m d h \Delta s t p k:: m d h \Delta f k:: m d h$ and $\Delta d t s \Delta l d h \Delta p a t:: m d h \Delta s t p k::$ $m d h \Delta f k:: m d h$ were constructed.

The yield of the fermentation products of mannitol was measured and tabulated (Fig. 8). The experimental results show that the mannitol synthesis ability of multi-gene deficiency and $m d h$ gene knock-in strains was increased compared with double-gene deficiency and $m d h$ gene knock-in strains. Among these, the highest mannitol productivity was with the multi-gene deficiency and $m d h$ gene knock-in strain $\Delta d t s \Delta l d h \Delta p a t:: m d h \Delta s t p k:: m d h \Delta f k:: m d h$, where the mannitol productivity reached $97.3 \%$ (increased by $24.7 \%$ over the wild strain).

\section{Discussion}

In order to facilitate screening of mutant strains, antibiotic resistance genes are often used as marker genes [28]. To avoid construction of antibiotic-resistant mutant strains, Zhang et al. [19] used the overlap extension to splice homologous fragments. Then the culture medium was supplemented with sucrose to screen for strains with low dextran yield for PCR confirmation. However, noantibiotic-resistance results in difficulty when screening for inactivated mutants. To solve this problem, a two-step homologous recombination method was used in this study to obtain markerless gene deficiency and gene knock-in strains. In this method, the $\alpha$-amy expression cassette was used as a marker gene.

Liu X. et al. [29] screened on $\mathrm{pH} 4.5$ agar plates with starch as the sole carbon source and observation of the $\mathrm{I}_{2}$ starch clear zone surrounding the colonies. However, $\mathrm{I}_{2} / \mathrm{KI}$ mix solution cannot be sterilized with the culture medium except being sprayed on the plate, which leads to potential contamination of the strains. The study of Tian et al. [20] shows that when soluble starch and trypan blue were added to the agar plate, the L. mesenteroides CGMCC1.10327 mutant strain, which carried the $\alpha$-amy expression cassette, would adsorb the trypan blue in the medium with the increase of incubation time, that resulted in the strain becoming blue and the color of the medium becoming lighter. Eric et al. [30] isolated the $\alpha$-amy expression cassette from the Lactobacillus amyloliquefaciens at a length of $2,862 \mathrm{bp}$, which has five $273 \mathrm{bp}$ repeats at the $3^{\prime}$ end. However, Eric considered this repetition to be unrelated to amylase activity. Therefore, the $\alpha$-amy expression cassette sequence used in this study was 2,000 bp in length.

The transformants were incubated $3 \mathrm{~h}$, then the strains were diluted $10^{7}$ on eight agar MRS plates (which included trypan blue and soluble starch), in which the proportion of the target transformant was 1:160. The mutant strains with the $\alpha$-amy marker gene were obtained by electrical transformation with the wild-type strain as receptor bacteria, of which the color of target transformants was blue when incubated for $5 \mathrm{~d}$ on agar MRS medium (the color of the wild-type strain is white). The color of the markerless mutant strains and $m d h$ knock-in mutant strains was white. It is good to be reminded that strains were obtained by electrical transformation with the mutant strains (which carried the $\alpha$-amy marker gene) as receptor bacteria.

Zhang et al. [19] knocked out the dts (dextransucrase) gene and the mannitol productivity was increased by $15.3 \%$ over the wild strain. Tian et al. [20] knocked out the ldh (lactate dehydrogenase) gene and the mannitol productivity was increased by $7 \%$ over the wild strain. Jin et al. (China patent CN 106754555A) knocked out the aldh (aldehyde dehydrogenase) gene and the mannitol productivity was increased by $19.8 \%$. In this study, acetate production increased while there was no significant change in lactate production. This result shows that knock-out of the pat gene results in the accumulation of acetyle phosphate, thus leading the carbon flux more toward the direction of acetate. This step was also accompanied by the production of ATP, which promotes the growth of the 
strain, thereby resulting in a more pronounced mannitol yield increase. This study found that multi-gene knockout has significant effect on mannitol production compared with single-gene knockout. $m d h$ gene knock-in increased the productivity of mannitol, of which the highest mannitol productivity was reached with multi-gene deficiency and $m d h$ gene knock-in strains (where the mannitol productivity reached $97.3 \%$ increased by $24.7 \%$ over the wild strain).

In conclusion, whether with gene knock-out or gene knock-in, we have reached the expected hypothesis. The study was based on the redirection of carbons toward the production of byproducts, and led to the development of Leuconostoc strains with high efficiency for mannitol production. Therefore, mannitol production was dramatically improved with knock-out of pat, $f k$, stpk and knock-in of $m d h$. The highest mannitol production was with multi-gene deficiency and $m d h$ gene knock-in strain $\Delta d t s \Delta l d h \Delta p a t:: m d h \Delta s t p k:: m d h \Delta f k:: m d h$, where the mannitol productivity reached $97.3 \%$ (increased by $24.7 \%$ over the wild strains).

\section{Conflict of Interest}

The authors have no financial conflicts of interest to declare.

\section{References}

1. Papagianni M, Legiša M. 2014. Increased mannitol production in Lactobacillus reuteri ATCC 55730 production strain with a modified 6-phosphofructo-1-kinase. J. Biotechnol 181: 20-26.

2. Wisselink HW, Weusthuis RA, Eggink G, Hugenholtz J, Grobben GJ. 2002. Mannitol production by lactic acid bacteria: a review. Int. Dairy J. 12: 151-161.

3. Makkee M, Kieboom APG, Van Bekkum H. 1985. Production methods of D-mannitol. Starch-Stärke 37: 136-141.

4. Dai Y, Meng Q, Mu W, Zhang T. 2017. Recent advances in the applications and biotechnological production of mannitol. J. Funct. Foods 36: 404-409.

5. Saha BC, Racine FM. 2011. Biotechnological production of mannitol and its applications. Appl. Microbiol. Biotechnol. 89: 879-891.

6. Wang Y. 2006. The production of mannitol by biotechnics (In Chinese). Mod. Food Sci. Technol. 22: 291-293.

7. Carvalheiro F, Moniz P, Duarte LC, Esteves MP \& Gírio FM. 2011. Mannitol production by lactic acid bacteria grown in supplemented carob syrup. J. Ind. Microbiol. Biotechnol. 38: 221-227.

8. Ghoreishi SM, Shahrestani RG. 2009. Innovative strategies for engineering mannitol production. Trends Food Sci. Technol. 20: 263-270.
9. Slatner M, Nagl G, Haltrich D, Kulbe KD, \& Nidetzky B. 1998. Enzymatic production of pure D-mannitol at high productivity. Biocatal. Biotransform. 16: 351-363.

10. Tomaszewska L, Rywińska A, Gładkowski W. 2012. Production of erythritol and mannitol by Yarrowia lipolytica yeast in media containing glycerol. J. Ind. Microbiol. Biotechnol. 39: 1333-1343.

11. Von Weymarn N, Hujanen M, Leisola M. 2002. Production of D-mannitol by heterofermentative lactic acid bacteria. Process Biochem. 37: 1207-1213.

12. Chung $\mathrm{CH}$. 2006. Production of glucooligosaccharides and mannitol from Leuconostoc mesenteroides B-742 fermentation and its separation from byproducts. J. Microbiol. Biotechnol. 16: 325-329.

13. Saha BC, Racine FM. 2008. Production of mannitol by lactic acid bacteria: a review. pp. 391-404. In Ching T Hou, Jei-Fu Shaw (eds.), Biocatalysis and Bioenergy. Wiley, Hoboken.

14. Song SH, Vieille C. 2009. Recent advances in the biological production of mannitol. Appl. Microbiol. Biotechnol. 84: 55-62.

15. Grobben GJ, Peters SW, Wisselink HW, Weusthuis RA, Hoefnagel MH, Hugenholtz J, et al. 2001. Spontaneous formation of a mannitol-producing variant of Leuconostoc pseudomesenteroides grown in the presence of fructose. Appl. Environ. Microbiol. 67: 2867-2870.

16. Saha BC. 2006. A low-cost medium for mannitol production by Lactobacillus intermedius NRRL B-3693. Appl. Microbiol. Biotechnol. 72: 676-680.

17. Fontes CP, Honorato TL, Rabelo MC, Rodrigues S. 2009. Kinetic study of mannitol production using cashew apple juice as substrate. Bioprocess Biosyst. Eng. 32: 493-499.

18. Yue M, Cao H, Zhang J, Li S, Meng Y, Chen W, et al. 2013. Improvement of mannitol production by Lactobacillus brevis mutant 3-A5 based on dual-stage $\mathrm{pH}$ control and fed-batch fermentations. World J. Microbiol. Biotechnol. 29: 1923-1930.

19. Zhang Z, Cheng WY, Ju XY, Jin HX. 2015. The effect of dextransucrase gene inactivation on mannitol production by Leuconostoc mesenteroides. Indian J. Microbiol. 55: 35-40.

20. Tian Y-F, Liu X-L, Cheng W-Y, Jin H-X. 2016. Study on application of $\alpha$-amylase gene in the gene expression of Leuconostoc mesenteroides (In Chinese). Sci. Technol. Food Ind. 37: 203-207.

21. Vingataramin L, Frost EH. 2015. A single protocol for extraction of gDNA from bacteria and yeast. Biotechniques 58: 120-125.

22. Gänzle MG. 2015. Lactic metabolism revisited: metabolism of lactic acid bacteria in food fermentations and food spoilage. Curr. Opin. Food Sci. 2: 106-117.

23. Helanto M, Aarnikunnas J, von Weymarn N, Airaksinen U, Palva A, Leisola M. 2005. Improved mannitol production by a random mutant of Leuconostoc pseudomesenteroides. J. Biotechnol. 116: 283-294.

24. Stancik IA, Šestak MS, Ji B, Axelson-Fisk M, Franjevic D, Jers C, et al. 2018. Serine/Threonine protein kinases from 
bacteria, archaea and eukarya share a common evolutionary origin deeply rooted in the tree of life. J. Mol. Biol. 430: 27-32.

25. Yan M-H, Wu Z-J. 2016. Proteomic analysis of EPS synthesis of Leuconostoc mesenteroides (In Chinese). Sci. Food Technol. Ind. 37: 158-160.

26. Chambel L, Chelo IM, Zé-Zé L, Pedro LG, Santos MA, Tenreiro R. 2006. Leuconostoc pseudoficulneum sp. nov., isolated from a ripe fig. Int. J. Syst. Evol. Microbiol. 56: 1375-1381.

27. Vidal RF, Martínez A, Moulis C, Escalier P, Morel S, Remaud-Simeon M, et al. 2011. A novel dextransucrase is produced by Leuconostoc citreum strain B/110-1-2: an isolate used for the industrial production of dextran and dextran derivatives. J. Ind. Microbiol. Biotechnol. 38: 1499-1506.
28. Guo X, Cao C, Wang Y, Li C, Wu M, Chen Y, et al. 2014 Effect of the inactivation of lactate dehydrogenase, ethanol dehydrogenase, and phosphotransacetylase on 2, 3-butanediol production in Klebsiella pneumoniae strain. Biotechnol. Biofuels 7: 44.

29. Liu X, Jia W, An Y, Cheng K, Wang M, Yang S, et al. 2015. Screening, gene cloning, and characterizations of an acidstable $\alpha$-amylase. J. Microbiol. Biotechnol. 25: 828-836.

30. Giraud E, Cuny G. 1997. Molecular characterization of the $\alpha$-amylase genes of Lactobacillus plantarum A6 and Lactobacillus amylovorus reveals an unusual 3' end structure with direct tandem repeats and suggests a common evolutionary origin. Gene. 198: 149-157. 